\title{
Can transcutaneous bilirubinometry reduce the need for blood tests in jaundiced full term babies?
}

\author{
L Briscoe, S Clark, C W Yoxall
}

Arch Dis Child Fetal Neonatal Ed 2002;86:F190-F192

Background: Previous studies have suggested that transcutaneous bilirubinometry (TCB) may provide a useful method for screening for significant jaundice, thereby reducing unnecessary blood tests. These studies have not allowed an estimation of the magnitude of such a benefit.

Objectives: To evaluate the accuracy of $T C B$ as a method of determining the need for serum bilirubin (SBR) measurements in full term babies and to quantify the magnitude of any benefit.

Subjects: Babies born at more than 34 weeks gestation who had not previously been exposed to phototherapy and were requiring blood sampling in the first week of life.

See end of article for authors' affiliations

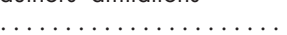

Correspondence to: Dr Yoxall, Liverpool Womens Hospital, Crown Street, Liverpool L8 7SS,

UK; Bill.Yoxall@

Iwh-tr.nwest.nhs.uk

Accepted

22 November 2001
Method: TcB measurements were made at the same time as blood sampling. SBR was measured in all blood samples. For jaundiced babies, the ability of TcB to detect significant jaundice (SBR > 249 umol/l) was evaluated.

Results: There was a correlation between SBR and TcB measurements $(n=303, r=0.76$, $p<0.0001)$, but the $95 \%$ prediction interval for SBR from TcB was wide $( \pm 88.3 \mu \mathrm{mol} / \mathrm{l})$. For the 285 jaundiced babies, the area under the receiver operator characteristic curve was 0.89 . A TcB value of 18 detected significant jaundice with a sensitivity of $100 \%$ and a specificity (95\% confidence interval) of $45 \%(39 \%$ to $51 \%)$. If blood samples had only been taken from babies with a TcB value greater than 18 , the number of samples taken would have been reduced by $34 \%$.

Conclusions: SBR cannot be measured accurately by TcB. However, TcB measurements can be used to determine the need for blood sampling in jaundiced babies and will reduce the number of blood samples taken. Recent improvements in TCB may improve the performance of this method.
$\mathrm{T}$ he decision to measure serum bilirubin (SBR) in a jaundiced baby is subjective and depends on the junior doctor or midwife making a clinical assessment of the colour of the baby's skin. Physiological jaundice is of course common; $65 \%$ of babies have SBR concentrations of $85 \mu \mathrm{mol} / \mathrm{l}$ or more, with the peak concentration occurring on day 5 of life, ${ }^{1}$ but only $6 \%$ of newborn infants develop hyperbilirubinaemia $(>220 \mu \mathrm{mol} / \mathrm{l}){ }^{2}$ There is a significant error in the clinical assessment of neonatal jaundice, and many of the measurements made are below the threshold for treatment.

Measuring SBR in babies with a concentration below the treatment threshold involves unnecessary blood sampling. The wait for the result may also unnecessarily delay discharge of both the mother and baby from the hospital. Transcutaneous bilirubinometry (TcB), using the Minolta Air Shields jaundice meter JM-102 (Hill-Rom-Airshields, Ashby de la Zauch, UK), has been proposed as a method of determining whether a baby requires SBR to be measured. It is a non-invasive optical method, which assesses jaundice by measuring the absorbance of reflected light in the blue and green wavebands to measure the "yellowness" of the skin. ${ }^{3}$

Several publications have described TcB as a useful screening method for detecting clinically significant jaundice. Strange and Cassady ${ }^{4}$ reviewed all such studies before 1985 and reported sensitivity values ranging from $83 \%$ to $100 \%$, with specificity values of 34-97\%. Most of these studies were flawed by the inclusion of multiple TcB measurements from some infants. In addition, all of these studies were small, with a range of $8-129$ total measurements. The total number of measurements made in babies with clinically significant bilirubin concentrations in each study was very small, and this also reduces the value of each of these studies as an evaluation of TcB as a screening method. Similar studies have been reported since $1985^{56}$ with similar results and similar limitations. Nonetheless, one American group has reported a significant reduction in SBR measurements made in their institution since the introduction of TCB measurements. ${ }^{7}$

Suckling et $a l^{8}$ reported the use of the bilirubinometer to identify babies with hyperbilirubinaemia in a UK population of full term babies. Unfortunately, in that study, SBR was only measured in those babies who had a TcB measurement above the threshold value, and therefore the false negative rate could not be determined.

To define the most effective cut off value for a screening test, it is necessary to construct a receiver operator characteristic (ROC) curve. Lin et $a l^{9}$ have done this for TcB in a Chinese population and reported that the best performance they could achieve was a sensitivity of $84 \%$ with a specificity of $84 \%$.

The primary aim of our study was to develop an ROC curve for TcB measurement to detect hyperbilirubinaemia in term babies in the first week of life, and also to identify the most informative value of TcB in terms of sensitivity and specificity. We also wished to estimate the magnitude of the reduction in SBR measurements in our hospital that we could achieve by adopting TcB as a screening method in term babies with clinically detectable jaundice.

\section{METHODS}

This prospective observational study was carried out at Liverpool Womens Hospital, a regional teaching hospital with about 6000 deliveries per annum.

Babies on the postnatal wards with a gestation of more than 34 weeks who were having blood taken for any reason were eligible to enter the study unless they had previously received

Abbreviations: $S B R$, serum bilirubin; $T c B$, transcutaneous bilirubinometry 


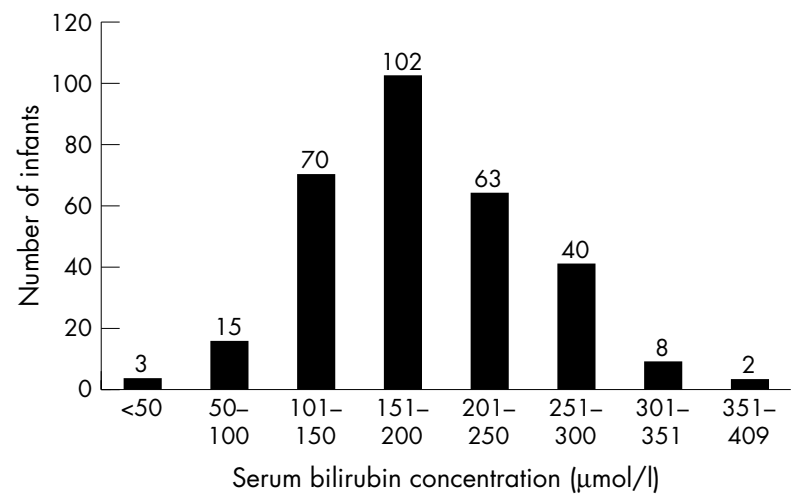

Figure 1 Distribution of serum bilirubin measurements.

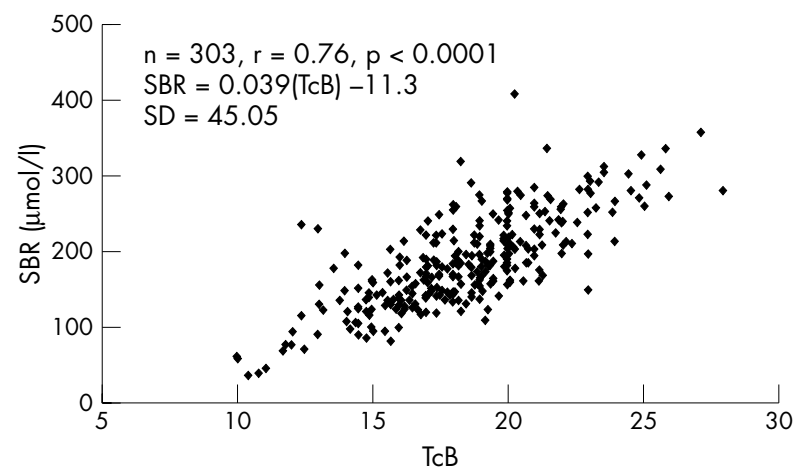

Figure 2 Correlation between transcutaneous bilirubinometry (TcB) and serum bilirubin (SBR) measurements.

phototherapy. Most of the babies were having blood taken for SBR measurement to evaluate clinical jaundice, but some non-jaundiced babies were also included. Measurements from non-jaundiced babies were used to investigate the correlation between the two methods, but not for assessing the effectiveness of TcB as a screening test. Because skin coloration is known to affect the TcB measurement, the ethnic origin of each baby was recorded.

After informed written consent was obtained from the parents, blood was taken for SBR. TcB measurements were made by the phlebotomist concurrently with the blood test. The mean of three TcB measurements from the baby's forehead was taken. Each eligible infant had only one paired measurement.

SBR was measured in the laboratory using a standard diazo method (Cobas Integra 700; Roche Diagnostics, Welwyn Garden City, Herts, UK). This method is known to be prone to interference by high haemoglobin concentrations, but this becomes less significant at higher bilirubin concentrations. This method is therefore not ideal for measuring SBR, but these measurements are those used by staff to determine management of jaundiced babies in everyday clinical practice. This was a pragmatic study to determine whether TcB measurements would alter clinical practice.

The indication for starting phototherapy in this group of babies in our hospital is an SBR concentration of $\geqslant 250 \mu \mathrm{mol} / \mathrm{l}$ on the second day of life, or $\geqslant 300 \mu \mathrm{mol} / \mathrm{l}$ thereafter. For the purposes of analysis, the definition of hyperbilirubinaemia was an SBR concentration $>249 \mu \mathrm{mol} / \mathrm{l}$.

\section{Sample size}

The number of babies needed to produce an adequate ROC curve was estimated using the method of Schafer. ${ }^{10}$ This was an estimate because of the paucity of data available to allow calculation of a true sample size. Assuming a "true" sensitiv-

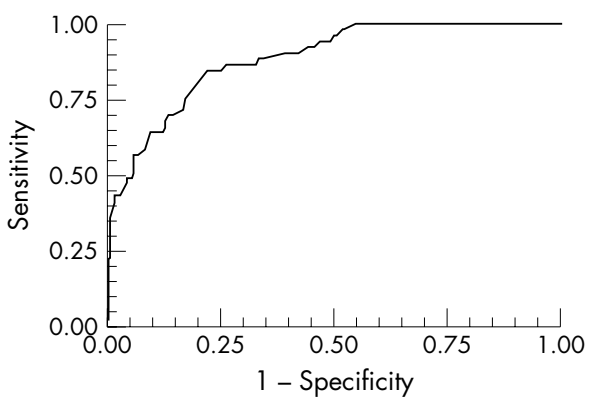

Figure 3 Receiver operator characteristics curve of transcutaneous bilirubin to detect serum bilirubin $>249 \mu \mathrm{mol} / \mathrm{l}$.

ity of $95 \%, \alpha=0.05, \beta=0.1, \rho=1$, and a minimum required sensitivity of $90 \%$, a total of 452 babies was required.

\section{Analysis}

The relation between TcB values and all SBR measurements was investigated using simple linear regression analysis.

A ROC curve was constructed to determine which TcB value had the greatest overall predictive power in babies in whom the blood test had been performed to evaluate clinical jaundice. The lowest TcB value to give 100\% sensitivity for detecting significant jaundice in these babies was also determined, and the effect of using this value on the number of SBR measurements was determined.

\section{RESULTS}

Consent was obtained for a total of 469 babies to enter the study. Paired measurements were available for only 303 babies. In the other 166 babies, paired data were not available because the decision to measure SBR was cancelled $(n=69)$, because an inadequate sample of blood was collected for SBR measurement $(\mathrm{n}=49)$, or because the bilirubinometer was not available $(n=48)$. Although we had not obtained our intended number of paired measurements, recruitment into the study was terminated on the basis of an interim analysis of the results at that point which showed that our aims had been achieved. In 285 of the 303 babies with paired measurements, SBR was measured to evaluate their jaundice, and, in the other 18 babies, SBR was measured in blood samples taken for other purposes.

The median (range) values for birth weight, gestation, and age were 3267 (1800-5008) g, 39 (34-42) weeks, and 3 (0-13) days respectively. Most (287) of the subjects were white. This is representative of the total population within our hospital, where $94 \%$ of the babies born are white.

The SBR concentrations were normally distributed (fig l). Most measurements fell between 150 and 300 pmol/l. Eighteen babies had concentrations below $100 \mu \mathrm{mol} / \mathrm{l}$, and 10 had concentrations above $300 \mu \mathrm{mol} / \mathrm{l}$.

There was a significant correlation between SBR and TcB measurements ( $\mathrm{n}=303, r=0.76, \mathrm{p}<0.0001$; fig 2 ). The prediction interval for SBR from TCB measurement was wide, however. The standard deviation about the regression line was $45.05 \mu \mathrm{mol} / \mathrm{l}$, giving a $95 \%$ prediction interval of $\pm 88.3 \mu \mathrm{mol} / \mathrm{l}$.

Of the 285 babies for whom SBR was measured to evaluate clinically apparent jaundice, 53 had significant jaundice (SBR $>249 \mu \mathrm{mol} / \mathrm{l}$ ). The area under the ROC curve was 0.89 (fig 3). Analysis of the ROC curve showed that the TcB value that detected significant jaundice (SBR > $249 \mu \mathrm{mol} / \mathrm{l}$ ) with the greatest predictive value was 19.9. This gave a sensitivity (95\% confidence interval (CI)) of $86 \%$ (81\% to $89 \%$ ) and a specificity ( $95 \%$ CI) of $78 \%$ ( $73 \%$ to $83 \%$ ). However, for clinical purposes, this is unsatisfactory as a sensitivity of $100 \%$ is needed. The TcB value that gave $100 \%$ sensitivity was 18 , which gave a specificity ( $95 \% \mathrm{CI})$ of $45 \%$ (39\% to $51 \%$ ). 
In our cohort of 285 babies having blood tests to evaluate their jaundice, if we had taken blood from only babies with a meter reading of 18 or more, we would have prevented 104 blood tests, a reduction of $34 \%$.

\section{DISCUSSION}

Although there is a close correlation between TcB and SBR measurements, the prediction interval for SBR from TcB is wide, and TcB cannot be used to measure SBR. However, the relation between TCB values and SBR allows TcB to be used as a screening tool for babies requiring SBR measurement.

In our unit, if TcB measurements were used to determine the need for SBR measurements in babies in whom there is clinical concern about significant jaundice, then the number of blood tests would be reduced by $34 \%$. It should be remembered that the predictive ability of any test is reduced when it is applied to a population with a lower prevalence of the condition being tested for. The use of TcB as a screening test in babies in whom there is no clinical concern about jaundice cannot therefore be justified on the basis of this study. Furthermore, because of differences in population characteristics and laboratory methods of assaying bilirubin, the manufacturers of the transcutaneous bilirubinometer recommend that each unit should develop a cut off point for use with this instrument that is appropriate for its population. The results of our study are therefore not directly transferable to other units. The babies in this study were mostly white. In a population with a higher proportion of non-white babies, the performance of TcB measurements is unlikely to give the same performance, as skin pigmentation is known to affect the TcB reading. ${ }^{6} 11$

Recent developments in the field of TcB include the development of newer bilirubinometers using more sophisticated optical technology, which may allow measurement of bilirubin rather than simply measuring skin "yellowness". ${ }^{12}{ }^{13}$ These may eventually prove to be more accurate methods for non-invasive screening for jaundice, but further published data are awaited.

It could be argued that TcB is a better method of measuring jaundice than SBR as it measures tissue bilirubin deposition rather than concentration in the blood. Unfortunately, there are no epidemiological data to support the use of TcB rather than SBR measurements. The relation between bilirubin concentration in the skin and in the central nervous system is not known, so the value of TCB in assessing tissue bilirubin concentration on the other side of the blood-brain barrier also remains unknown. In reality, most paediatricians are likely to adopt a pragmatic approach and rely on SBR measurements, with which there has been extensive experience developed over many years.

There is no consensus about when to evaluate and treat neonatal jaundice. Most of the scientific data relating to "safe" levels of hyperbilirubinaemia have been obtained from uncontrolled observations of babies with haemolytic disease of the newborn. However, these observations were largely made over 30 years ago when many of the babies had associated prematurity and asphyxia and were often exposed to the antibiotic streptomycin. All of these factors are known to potentiate bilirubin toxicity, and the "safe" level of bilirubin for haemolytic disease in the modern era is not clearly known. There is also a paucity of scientific evidence as to the safe level of bilirubin in healthy term babies without haemolysis. Despite this, most neonatal authorities believe that there is still a risk to term babies with non-haemolytic jaundice and, although there is no clear consensus on a definition, all would recommend treatment of hyperbilirubinaemia.

Treatment of jaundice will remain a common intervention in neonatal medicine, and this will be based on SBR measurements. Clinical assessment of jaundice is inaccurate and results in a significant number of blood tests in otherwise well babies. The number of these blood tests could be reduced, with benefit to the babies and potential cost savings, by using TcB as a screening tool

\section{Authors' affiliations \\ L Briscoe, S Clark, C W Yoxall, Liverpool Womens Hospital, Crown Street, Liverpool L8 7SS, UK}

\section{REFERENCES}

1 Maisels MJ. Neonatal jaundice. In: Sinclair JC, Bracken MB, eds. Effective care of the newborn. Oxford: Oxford University Press, 1993:507-562.

2 Dai J, Parry DM, Krahn J. Transcutaneous bilirubinometry: its role in the assessment of neonatal jaundice. Clin Biochem 1997;30:1-9.

3 Yamamuchi Y, Yamanishi A, Yamanouchi I. Transcutaneous monitoring of bilirubin Minolta jaundice meter. In: Brans YW, Hay WW, eds. Physiological monitoring and instrument diagnosis in perinatal and neonatal medicine. Cambridge: Cambridge University Press, 1995.

4 Strange $M$, Cassady G. Neonatal transcutaneous bilirubinometry. Clin Perinatol 1985;12:51-62.

5 Schumacher RE, Thornberry JM, Gutcher GR. Transcutaneous bilirubinometry: a comparison of old and new methods. Pediatrics 1985;76:10-14.

6 Linder N, Regev A, Gazit G, et al. Noninvasive determination of neonatal hyperbilirubinaemia: standardisation for variation in skin color. Am J Perinatol 1994:3:223-5.

7 Maisels MJ, Kring E. Transcutaneous bilirubinometry decreases the need for serum bilirubin measurements and saves money. Pediatrics 1997;99:599-601.

8 Suckling RJ, Laing IA, Kirk JM. Transcutaneous bilirubinometry as a screening tool for neonatal jaundice. Scot Med J 1995:40:14-15.

9 Lin YJ, Ju SN, Lin CH. The clinical application of transcutaneous bilirubinometry in full term Chinese infants. Acta Paediatr Sin 1993;34:69-76.

10 Schafer, $\mathrm{H}$. Constructing cut-off point for a quantitative diagnostic test. Stat med 1989;8:1381-91.

11 Goldman SL, Penalver A, Penaranda R. Jaundice meter: evaluation of new guidelines. J Pediatr 1982;101:253-6.

12 Tayabe R, Gribetz D, Gribetz I, et al. Noninvasive estimation of serum bilirubin. Pediatrics 1988;102:E28.

13 Bhutani VK, Gourley GR, Adler SA, et al. Noninvasive measurement of total serum bilirubin in a multiracial predischarge newborn population to assess the risk of severe hyperbilirubinaemia. Pediatrics 2000;106:e17. 